\title{
Studies on the Inclusion Behavior of Amphiphilic p-Sulfonatocalix[4]arene with Ascorbic Acid by Spectrofluorometric Titrations
}

\author{
Yunyou Zhou, Xueping Ding, Xiaoli Fang, Tao Li, Dongbao Tang, Qin Lu \\ Key Laboratory of Chemo-Biosensing, College of Chemistry and Materials Science, Anhui Normal University, Wuhu, \\ China \\ E-mail: zy161299@mail.ahnu.edu.cn. \\ Received April 5, 2011; revised May 6, 2011; accepted May 16, 2011
}

\begin{abstract}
The aqueous solution of tetrabutyl ether derivatives of $p$-sulfonatocalix[4]arene (SC4Bu) and ascorbic acid (AA) complex has been studied based on fluorescence and ${ }^{1} \mathrm{H}$ NMR spectroscopic results. It was found that the fluorescence intensity of SC4Bu quenched regularly upon the addition of AA. A 1:1 stoichiometry for the complexation was established and was verified by Job's plot. The temperature-dependent inclusion constants were calculated, form which $\Delta H$ and $\Delta S$ values were calculated. Meanwhile the proposed interaction mechanism of the inclusion complex was discussed based on ${ }^{1} \mathrm{H}$ NMR results. The various factors (ionic strength, and surfactants) effecting the inclusion process were examined in detail.
\end{abstract}

Keywords: $P$-Sulfonatocalix[4]arene, Ascorbic Acid, Fluorescence Spectrometry, Inclusion Interaction

\section{Introduction}

Calixarenes and their derivatives have attracted considerable attention in host-guest chemistry due to their excellent recognition ability $[1,2]$. The water-soluble $p$-sulphonated calyx $[n]$ arenes $(\mathrm{SC} n \mathrm{~A}, n=4,6,8)$ derivatives display wider applications in supramolecular chemistry science because they allow the study of host-guest interactions in a solvent where most biological processes take place [3-9]. $P$-sulphonated calix[ $n$ ] arenes (SCnA, $n$ $=4,6,8)$ have been reported to interact with specific drugs or their intermediates [10-12]. However, SC4A and SC6A have very weak fluorescence and absorption signals in an aqueous solution [10]. They are difficult to be used for the investigation of inclusion behavior of non-fluorescent or weakly fluorescent guest molecules [13]. Therefore, it is of great importance to synthesize fluorescent water-soluble calixarene derivatives for studying the inclusion behavior of these non-fluorescent molecules.

Ascorbic acid (AA, Scheme 1), commonly known as Vitamin $\mathrm{C}$, is one of the most important water-soluble vitamins in foods and drinks [14,15]. It is also widely used as a food additive and antioxidant [16], which can prevent scurvy and reduce the incidence and mortality from cardiovascular disease and cancer [17].

Herein, we have synthesized the tetrabutyl ether derivative of p-sulfonatocalix[4] arene ( $\mathrm{SC} 4 \mathrm{Bu}$, Scheme 1) with strong fluorescence in aqueous solution and also investigated the inclusion behavior of $\mathrm{SC} 4 \mathrm{Bu}$ with $\mathrm{AA}$ in aqueous solution using fluorescence spectrometry. The results indicate that $\mathrm{SC} 4 \mathrm{Bu}$ and $\mathrm{AA}$ form a complex (1:1 mole ratio), with a binding constant of $1.78 \times 10^{3} \mathrm{~L} \cdot \mathrm{M}^{-1}$. The interaction mechanism of the inclusion process was discussed. This work may extend the application of $\mathrm{SC} 4 \mathrm{Bu}$ in the biochemistry area.

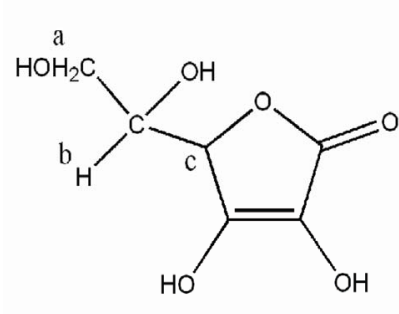

(1)

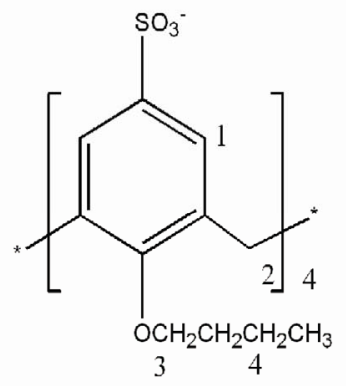

(2)
Scheme 1 chemical structure of ascorbic acid (1) and SC4Bu (2) 


\section{Experimental}

\subsection{Apparatus}

Fluorescence spectra and relative fluorescence intensities were performed on a model F-4600 fluorescence spectrophotometer (Hitachi, Japan) using a $1 \mathrm{~cm} \times 1 \mathrm{~cm}$ quartz cell. The slit widths for excitation and emission were both set to $5.0 \mathrm{~nm}$. The fluorescence lifetimes were measured on a FLS920 combined steady-state lifetime fluorescence spectrometer (Edinburgh Instrument). All measurements were carried out at desired temperature adjusted by a thermostatic cell holder. ${ }^{1} \mathrm{H}$ NMR was performed on an Avance Bruker-300 MHz spectrometer. IR was obtained using a PE983 infrared spectrophotometer (Perkin Elmer).

\subsection{Chemicals}

$\mathrm{SC} 4 \mathrm{Bu}$ was synthesized according to the literature [18, 19] and identified by IR and ${ }^{1} \mathrm{H}$ NMR. AA was purchased from Shanghai Chemical Reagent Co. All other reagents were of analytical reagent grade and used without any further purification. Doubly distilled water was used throughout.

\subsection{Procedure}

A $1.0 \mathrm{~mL}$ of $1.00 \times 10^{-4} \mathrm{~mol} \cdot \mathrm{L}^{-1} \mathrm{SC} 4 \mathrm{Bu}$, and an appropriate amount of $1.00 \times 10^{-3} \mathrm{~mol} \cdot \mathrm{L}^{-1}$ AA were transferred into a $10 \mathrm{~mL}$ volumetric flask. The mixture was diluted to the final volume with water and mixed thoroughly. Fluorescence spectra were measured at $25.0^{\circ} \mathrm{C}$.

\section{Results and Discussion}

\subsection{Characteristics of Fluorescence Spectra}

Figure 1 displays the fluorescence spectra of $1.00 \times 10^{-5}$ $\mathrm{mol} \cdot \mathrm{L}^{-1} \mathrm{SC} 4 \mathrm{Bu}$ in aqueous solution. It is clear that $\mathrm{SC} 4 \mathrm{Bu}$ shows strong fluorescence in aqueous solution with excitation and emission wavelengths at 230 and 315 $\mathrm{nm}$, respectively. When an appropriate amount of $1.00 \times$ $10^{-3} \mathrm{~mol} \cdot \mathrm{L}^{-1} \mathrm{AA}$ was added to $\mathrm{SC} 4 \mathrm{Bu}$, the fluorescence quenching of SC4Bu was observed, as shown in Figure $\mathbf{2}$, indicating the formation of the inclusion complexes.

The formation of inclusion complex was also revealed by the fluorescence decay curves obtained at different concentrations of AA. The fluorescence lifetimes of the SC4Bu molecule in aqueous and in AA were determined from the decay curves and the results are shown in Table 1. The fluorescence decay of $\mathrm{SC} 4 \mathrm{Bu}$ without AA obtained from monitoring the emission at $312 \mathrm{~nm}$ is a single-exponential decay with lifetime value of $0.72 \mathrm{~ns}\left(\tau_{1}\right)$.

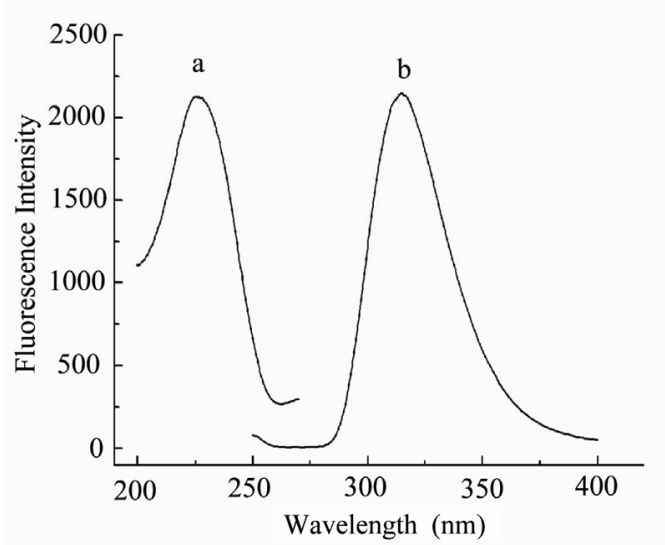

Figure 1. Fluorescence excitation (a) and emission (b) spectra of SC4Bu $\left(1.00 \times 10^{-5} \mathrm{~mol} \cdot \mathrm{L}^{-1}\right)$ in solution.

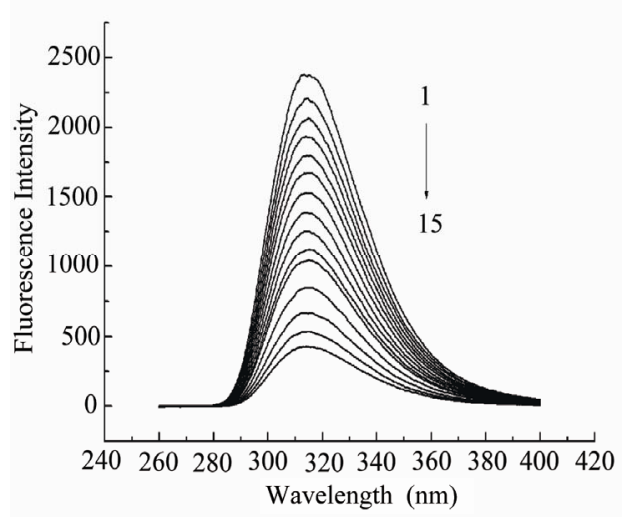

Figure 2. Fluorescence spectra of SC4Bu with different concentrations of AA. The concentrations from (1) to (15) of AA were: (1) $0.00,(2) 0.20,(3) 0.40$, (4) $0.60,(5) 0.80$, (6) 1.00 , (7) 1.20, (8) 1.40, (9) 1.60, (10) 1.80 , (11) 2.00 , (12) 2.50, (13) 3.00 , (14) 3.50 and (15) $4.00 \times 10^{-4} \mathrm{~mol} \cdot \mathrm{L}^{-1}$. SC4Bu $=$ $1.00 \times 10^{-5} \mathrm{~mol} \cdot \mathrm{L}^{-1}, \mathbf{2 5 . 0} \mathrm{C}$.

Table 1. Time-resolved fluorescence spectral data of SC4Bu in the absence and presence of different concentrations of AA $\left(\lambda \mathrm{ex}=250 \mathrm{~nm}, \lambda \mathrm{em}=310 \mathrm{~nm} ;[\mathrm{SC} 4 \mathrm{Bu}]=1.0 \times 10^{-5} \mathrm{M}\right)$

\begin{tabular}{ccccc}
\hline $\begin{array}{c}\text { Concentration of } \\
\text { AA (M) }\end{array}$ & $\begin{array}{c}\text { Lifetime } \\
(\mathrm{ns})\end{array}$ & $\begin{array}{c}\text { Relative ampli- } \\
\text { tudes }(\%)\end{array}$ & $\chi^{2}$ & $\begin{array}{c}\text { Standard devia- } \\
\text { tion (ns) }\end{array}$ \\
\hline 0 & 0.72 & 100 & 1.018 & 0.02 \\
$0.2 \times 10^{-5}$ & 0.78 & 84.12 & 1.062 & 0.02 \\
& 2.52 & 15.88 & & 0.16 \\
$0.4 \times 10^{-5}$ & 0.77 & 82.53 & \multirow{2}{*}{1.002} & 0.02 \\
& 2.62 & 17.47 & & 0.17 \\
$0.8 \times 10^{-5}$ & 0.76 & 81.86 & \multirow{2}{*}{1.021} & 0.02 \\
& 2.50 & 18.14 & & 0.14 \\
\hline
\end{tabular}

Upon the addition of AA, single exponential decay changes to biexponential decay with lifetime $\tau_{1}$ and $\tau_{2}$, respectively, suggesting the presence of two species, SC4Bu and its complex. It is also evident from Table 1 that the short-lived species $\left(\tau_{1}\right)$ is close to the measured lifetime of 
$\mathrm{SC} 4 \mathrm{Bu}$ in pure water, and that of the long-lived species $\tau_{2}$ are correspond to the inclusion complex. The amplitude of the complex form also increases due to an increase in complex formation and the free species decreasing.

\subsection{Stoichiometry and Inclusion Constant}

The formation of 1:1 SC4Bu-AA complex was confirmed by the continuous variation method (Job's plot) using fluorescence spectrometry. Here, the solutions of $\mathrm{SC} 4 \mathrm{Bu}$ and AA were mixed in different mole ratio keeping the total of the $\mathrm{SC} 4 \mathrm{Bu}$ and $\mathrm{AA}$ concentration constant. The maximum relative fluorescence intensity was observed when $[\mathrm{SC} 4 \mathrm{Bu}] /([\mathrm{SC} 4 \mathrm{Bu}]+[\mathrm{AA}])=0.5$ as shown in Figure 3, this is in agreement with the double reciprocal plot and confirmed the formation of a 1:1 inclusion complex in the system.

Assuming that $\mathrm{SC} 4 \mathrm{Bu}$ and $\mathrm{AA}$ forms a 1:1 ratio complex, according to our earlier work $[12,20]$ the equation of host-guest inclusion complex can be expressed as:

$$
\frac{1}{F-F_{0}}=\frac{1}{F_{1}-F_{0}}+\frac{1}{K[G]\left(F_{1}-F_{0}\right)}
$$

Here $F_{0}$ is the intensity of fluorescence of SC4Bu without AA, $F$ is the observed fluorescence intensity at different AA concentration tested, $F_{1}$ is the intensity with the highest concentration of AA and $K$ is the binding constant. The double reciprocal plots of $1 /\left[F-F_{0}\right]$ versus $1 /[\mathrm{AA}]$ are depicted in Figure 4. The plot displays a linear relation with a relative correlation constant of 0.9993 , indicating the formation of a 1:1 stoichiometry between SC4Bu and AA. The association constant $(\mathrm{K})$ obtained from the ratio of the intercept to the slope is $1.78 \times 10^{3} \mathrm{~L} \cdot \mathrm{M}^{-1}$.

\subsection{Thermodynamic Parameter Value}

The binding constants at various temperatures were investigated by measuring the fluorescence spectra of SC4Bu in the presence of AA, and the results were summarized in Table 2. By plotting $\operatorname{lnK}$ against $1 / \mathrm{T}$ (the Van't Hoff method) [19] (see Figure 5), the enthalpy $(\Delta H)$ and entropy $(\Delta S)$ were calculated to be $25.25 \mathrm{~kJ} \cdot \mathrm{mol}^{-1}, 146.75 \mathrm{~J}$ $\mathrm{mol}^{-1} \mathrm{~K}^{-1}$, respectively. These results suggested that the entropic driving force favored the formation of $\mathrm{SC} 4 \mathrm{Bu}-\mathrm{AA}$ complex. The relevant free energy change for this system is $\Delta G=-18.48 \mathrm{~kJ} \cdot \mathrm{mol}^{-1}$ at $298 \mathrm{~K}$, indicating that this inclusion process is an energetically favored process.

\section{4. ${ }^{1} \mathrm{H}$ NMR Studies}

Chemical shift variations of specific host or guest nucleus could provide evidence for the formation of inclusion complexes in solution, since significant changes in

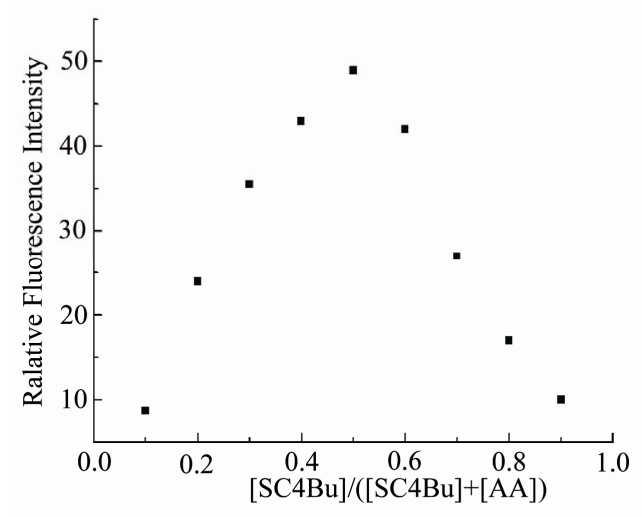

Figure 3. Job's plot of SC4Bu-AA system, [SC4Bu] + [AA] $=1.00 \times 10^{-5} \mathrm{~mol} \cdot \mathrm{L}^{-1}, 25.0^{\circ} \mathrm{C}$.

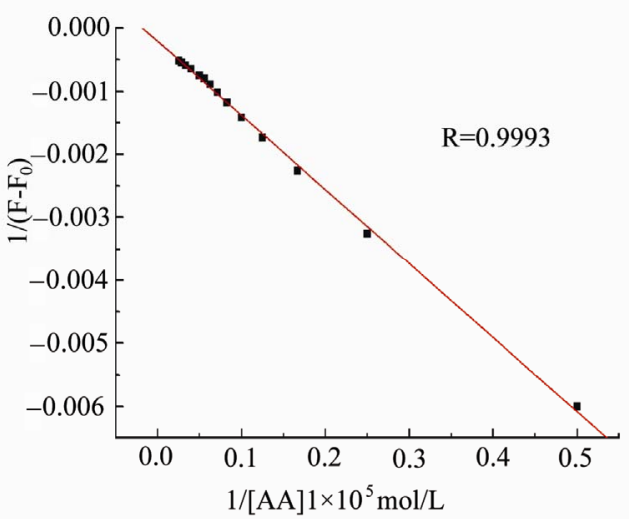

Figure 4. A plot of $1 /\left(F-F_{0}\right)$ versus $1 /[S C 4 B u]$ for $\mathrm{SC} 4 \mathrm{Bu}-$ AA complex.

Table 2. Inclusion constants of complexes at different temperatures.

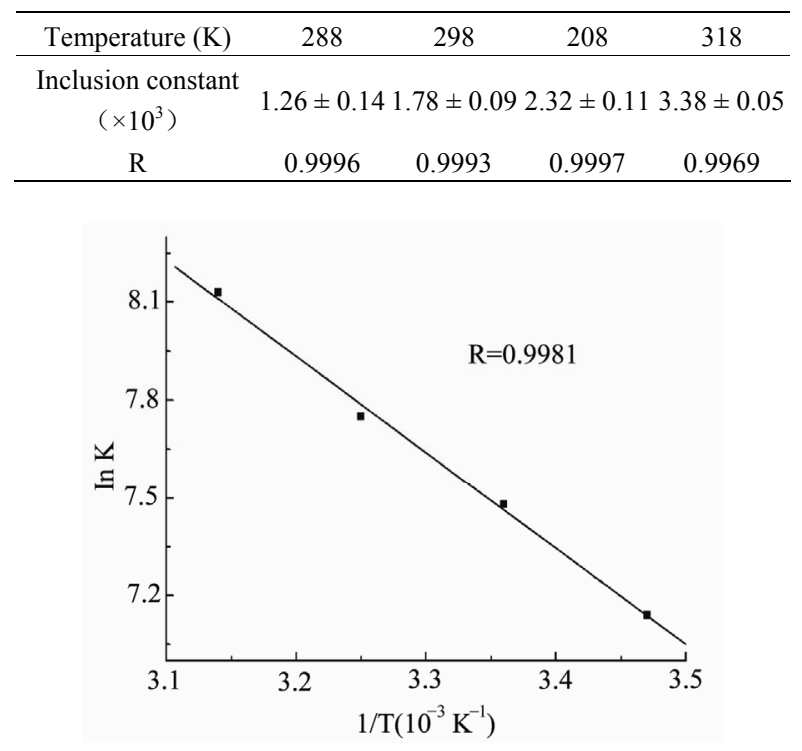

Figure 5. The plot of $\ln K$ versus $1 / T$ for the SC4Bu-AA complex. 
Table 3. Chemical shifts $\delta$ and $\Delta \delta$ of protons of $\mathrm{AA}$ in free guest and inclusion complex.

\begin{tabular}{cccccccc}
\hline & $\mathrm{H}_{1}$ & $\mathrm{H}_{2}$ & $\mathrm{H}_{3}$ & $\mathrm{H}_{4}$ & $\mathrm{H}_{\mathrm{a}}$ & $\mathrm{H}_{\mathrm{b}}$ & $\mathrm{H}_{\mathrm{c}}$ \\
\hline$(\mathrm{AA}) \delta$ & & & & & 3.7185 & 4.0281 & 4.9374 \\
$(\mathrm{SC} 4 \mathrm{Bu}) \delta$ & 7.6359 & $4.1250 / 3.4247$ & 3.9762 & 1.4676 & & & \\
$(\mathrm{SC} 4 \mathrm{Bu}-\mathrm{AA}) \delta$ & 7.5850 & $4.0354 / 3.3976$ & 3.9607 & 1.4657 & 3.7463 & 4.0354 & 4.9212 \\
$\delta \Delta$ & -0.0509 & $-0.0896 /-0.0271$ & -0.0155 & -0.0019 & +0.0278 & +0.0073 & -0.0162 \\
\hline
\end{tabular}

microenvironment are known to occur between the free and bound states [21]. The interaction between $\mathrm{SC} 4 \mathrm{Bu}$ and AA were studied by ${ }^{1} \mathrm{H}$ NMR spectra. The significant distinction between the ${ }^{1} \mathrm{H}$ NMR spectra of SC4Bu and the inclusion complex of AA with $\mathrm{SC} 4 \mathrm{Bu}$ in $\mathrm{D}_{2} \mathrm{O}$ confirms that the inclusion complex was formed and the value of chemical shifts for different protons in $\mathrm{SC} 4 \mathrm{Bu}, \mathrm{AA}$ and SC4Bu-AA inclusion complex as shown in Table 3.

The ${ }^{1} \mathrm{H}$ NMR shifts are in agreement with those reported in the literatures [21-22], which mentioned that downfield shifts should be observed for guest protons and upfield shifts for host protons upon hydrophobic interactions between both partners. The complexation causes an upfield shift of SC4Bu protons: a great upfield was recorded for $\mathrm{H}_{1}$ and $\mathrm{H}_{2}$. Since both of them are located inside the cavity. The upfield shift observed for $\mathrm{H} 2$ and $\mathrm{H} 1$ confirms the inclusion inside the cavity. Further confirmation was obtained by observing the changes in the chemical shifts of AA.

The H-chemical shifts in both the free and the complexed state are reported in Table 3. We can see that $\mathrm{H}_{\mathrm{c}}$ proton was shifted upfield, while $\mathrm{H}_{\mathrm{a}}$ and $\mathrm{H}_{\mathrm{b}}$ were deshielded upon complexation. In fact, the downfield shifts of the guest protons have been attributed to a variation of local polarity when these protons are inside the cavity [21]. The shielding of $\mathrm{H}_{\mathrm{c}}$ proton has been attributed to it is close to the cavity of SC4Bu. As expected, the probable binding mode in the $\mathrm{SC} 4 \mathrm{Bu}$ inclusion complexes involves the insertion of less part of the guest into the cavity and exposure of more groups to the bulk solvent outside.

\subsection{Discussion of Interaction Mechanism}

Calixarenes and their derivatives have been known to be able to form non-covalent inclusion complexes with various guest molecules through many interactions, such as the electrostatic interaction, cation $-\pi$ interactions, hydrogen bonding, van der Waals and hydrophobic interactions [23]. In this context, considering the size/shape fitting, multiple recognition and ${ }^{1} \mathrm{H}$ NMR results, pattern inclusion manner between $\mathrm{SC} 4 \mathrm{Bu}$ and $\mathrm{AA}$ was proposed as show in Figure 6. AA partially goes into the cavity of $\mathrm{SC} 4 \mathrm{Bu}$ with the help of hydrogen bonding, which formed by the hydroxyl groups of AA bonding with sulphonyl groups of $\mathrm{SC} 4 \mathrm{Bu}$, respectively.
The main driving force for the inclusion of AA by $\mathrm{SC} 4 \mathrm{Bu}$ was investigated. The electrostatic effect was studied by varying the ionic strength of $\mathrm{NaCl}$ and the results were shown in Figure 7. As can be see when the $\mathrm{NaCl}$ concentration is $0.01 \mathrm{~mol} \cdot \mathrm{L}^{-1}$, the fluorescence intensity of the SC4Bu-AA system had no significant changes compared with the absence of $\mathrm{NaCl}$ in the system. Thus the main driving force for inclusion of AA by $\mathrm{SC} 4 \mathrm{Bu}$ should not be electrostatic interaction. The hydrogen bonding and the hydrophobic interaction should play the main driving force for this inclusion process. This is different from what the literature suggests, in which electrostatic interaction was thought to play important part in the inclusion process [12].

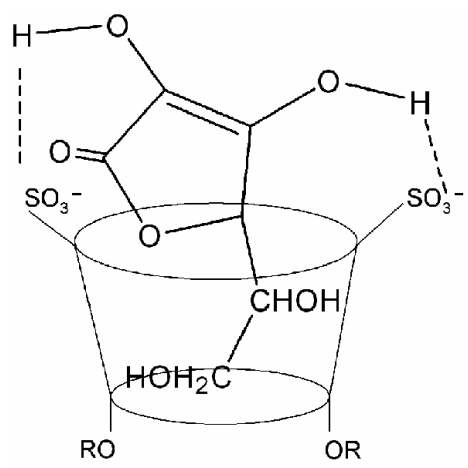

Figure 6. The proposed inclusion pattern of the SC4Bu-AA. complex.

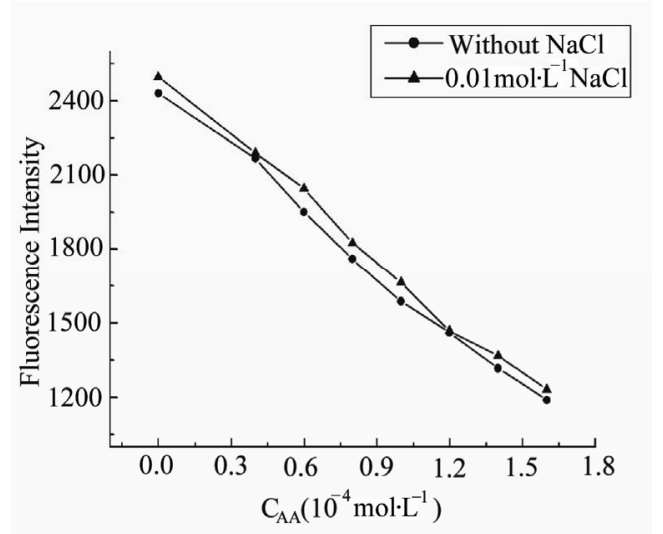

Figure 7. Influence ionic strength of $\mathrm{NaCl}$ on the fluorescence intensity of $\mathrm{SC} 4 \mathrm{Bu}-\mathrm{AA}$ system; $[\mathrm{SC} 4 \mathrm{Bu}]=1.00 \times 10^{-5}$ $\mathrm{mol} \cdot \mathrm{L}^{-1}, \mathrm{C}_{\mathrm{NaCl}}=0.01 \mathrm{~mol} \cdot \mathrm{L}^{-1}$. 


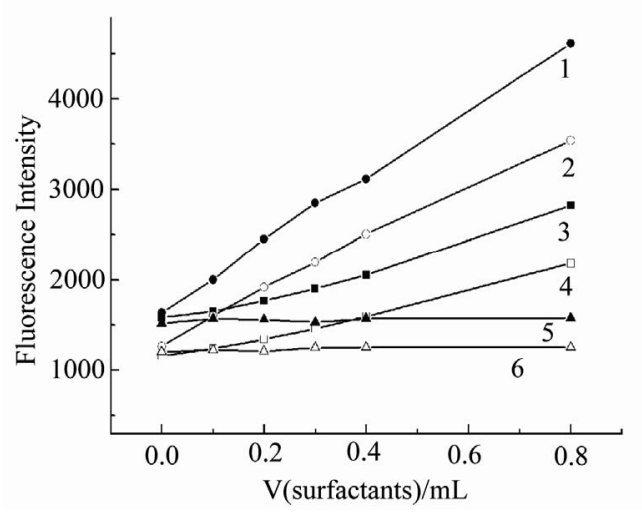

Figure 8. Influence of surfactants concentration on the fluorescence intensity of SC4Bu-AA system; (1) $\mathrm{SC} 4 \mathrm{Bu}+$ TritonX-100; (2) SC4Bu+AA+TritonX-100; (3) SC4Bu+CTAB; (4) SC4Bu+AA+ CTAB; (5) SC4Bu+SDS; (6) $\mathrm{SC} 4 \mathrm{Bu}+\mathrm{AA}+\mathrm{SDS} ;[\mathrm{SC} 4 \mathrm{Bu}]=5.00 \times 10^{-6} \mathrm{~mol} \cdot \mathrm{L}^{-1}$, $[\mathrm{AA}]$ $=6.00 \times 10^{-5} \mathrm{~mol} \cdot \mathrm{L}^{-1}$.

\subsection{Influence of Surfactants}

The influence of surfactants on this inclusion process was also investigated. Three types of surfactants: the cationic surfactant cetytrimethyammonium bromide (CTAB), the anionic surfactant sodium dodecyl sulfate (SDS) and the non-ionic surfactant octylophenylpolyoxyethylene ether (Triton X-100) were chosen to study their effect on SC4Bu and AA complex. Among those, SDS showed no influence on the fluorescence intensity of $\mathrm{SC} 4 \mathrm{Bu}$ and the SC4Bu-AA complex as shown in Figure 8, while Triton $\mathrm{X}-100$ and $\mathrm{CTAB}$ enhanced the fluorescence intensities of SC4Bu and the corresponding SC4Bu-AA complex. Triton-100 itself has been reported to be able to enhance the fluorescence intensity of certain types of sulfuric fluorophores [24], Similarly, it may also lead to the increase of the fluorescence intensity of $\mathrm{SC} 4 \mathrm{Bu}$ and the SC4Bu-AA complex. In our previous work, we have reported that SCnA can form a 1:1 complex with CTAB, which lead to the enhancement of the fluorescence intensity of SCnA [24]. Similarly, CTAB may lead to the increase of the fluorescence intensity of $\mathrm{SC} 4 \mathrm{Bu}$ through the inclusion process. Upon addition of AA, the quench of the fluorescence intensity of the SC4Bu-CTAB complex was observed, indicating the partial replacement of CTAB with AA [25].

\section{Conclusions}

In this work, a water-soluble $p$-sulfonatocalix[4]arene derivative $(\mathrm{SC} 4 \mathrm{Bu})$ with strong fluorescence was synthesized and inclusion process between $\mathrm{SC} 4 \mathrm{Bu}$ and $\mathrm{AA}$ in aqueous solution was also investigated by fluorescence spectrometry. AA and SC4Bu were found to form a 1:1 host-guest inclusion complex. The possible interaction mechanism was discussed. Similar to cyclodextrins, $\mathrm{SC} 4 \mathrm{Bu}$ and its inclusion complexes show potential for biological and medical applications.

\section{Acknowledgements}

The authors thank the financial supports of the National Natural Science Foundation of P. R. China (20875002).

\section{Reference}

[1] C. D. Gutsche, "Calixarenes Revisited: Monographs in Supramolecular Chemistry," Royal Society of Chemistry, Cambridge, 1998.

[2] J. H. Yang, F. Huang, M. Wang, X. Wu and C. X. Sun, "Study on the Molecular Recognitions of Calix $[n]$ arenes to 2,3-Diaminonaphthalene by Using Fluorometric Technique," Spectrochimica Acta Part A, Vol. 58, No. 8, 2002, pp. 1775-1778. doi:10.1016/S1386-1425(01)00629-1

[3] G. Arena, A. Contino, F. G. Gulino, A. Magri, F. Sansone, D. Sciotto and R. Ungaro, "Complexation of native L- $\alpha-$ Aminoacids by Water Soluble Calix[4]arenas," Tetrahedron Letters, Vol. 40, No. 8, 1999, pp. 1597-1600. doi:10.1016/S0040-4039(98)02654-9

[4] F. Sansone, S. Barboso, A. Casnati, D. Sciotto and R. Ungaro, Tetrahedron Letters, Vol. 40, No. 25, 1999, pp. 4741-4744. doi:10.1016/S0040-4039(99)00838-2

[5] K. M. Hwang, Y. M. Qi, S. Y. Liu, T. C. Lee, W. Choy and J. Chen, US Patent 5409 959, 1995.

[6] K. M. Hwang, Y. M. Qi, S. Y. Liu, W. Choy and J. Chen, US Patent 5441 983, 1995.

[7] J. L. Atwood, R. J. Bridges, R. K. Juneja, K. Ravindra and A. K. Singh, US Patent 748 764, 1996.

[8] D. J. S. Humes, A. W. Coleman and E. Aubert-Fouchet, French Patent FR 2782 007, 1998.

[9] E. Da Silva, P. Shahgaldian and A. W. Coleman, "Haemolytic Properties of Some Water-Soluble ParaSulphonato-Calix[n]arenas," Internationalt International Journal of Pharmaceutics, Vol. 273, No. 1-2, 2004, pp. 57-62. doi:10.1016/j.ijpharm.2003.12.008

[10] J. S. Millership, "A Preliminary Investigation Of The Solution Complexation of 4-Sulphonic Calix[n]arenas with Testosterone," Journal of Inclusion Phenomena and Macrocyclic Chemistry, Vol. 39, No. 3-4, 2001, pp. 327-331. doi:10.1023/A:1011196217714

[11] Y. Y. Zhou, Q. Lu, C. Liu, S. K. She and L. Wang, "Study on the Inclusion Behavior of p-Sulphon Atocalix[4]arene with 9-Amino-Acridine by Spectrofluore Metric Titrations," Spectrochim Acta Part A, Vol. 63, No. 2, 2006, pp. 423-426. doi:10.1016/j.saa.2005.04.056

[12] Y. Y. Zhou, Q. Lu, C. Liu, S. K. She and L. Wang, "A novel Spectrofluorimetric Method for Determination of Lomefloxacin Based on Supramolecular Inclusion Complex between it and p-Sulfonated Calix[4]arene," Ana- 
lytica Chimica Acta, Vol. 552, No. 1-2, 2005, pp. 152-159. doi:10.1016/j.aca.2005.07.038

[13] Q. Lu, J. S. Gu, H. P. Yu, C. Liu, L. Wang and Y. Y. Zhou, "Study on the Inclusion Interaction of $p$-Sulfonated Calix [ $n]$ arenas with Vitamin $\mathrm{K}_{3}$ Using Methylene Blue as a Spectral Probe," Spectrochimica Acta Part A, Vol. 68, No. 1, 2007, pp. 15-20. doi:10.1016/j.saa.2006.10.044

[14] C. Martin, "The Parkinson's Puzzle," Chemistry in Britain, Vol. 34, No. 99, 1998, pp. 40-42.

[15] A. Heinz, H. Przuntek, G. Winterer and A. Pietzcker, "Clinical Aspects and Follow-up of Dopamine-Induced Psychoses in Continuous Dopaminergic Therapy and Their Implications for the Dopamine Hypothesis of Schizophrenic Symptoms," Nervenarzt, Vol. 66, No. 9, 1995, pp. 662-669.

[16] J. Yang, R. Gadi, R. Paulino and T. Thomson, "Total Phenolics, Ascorbic Acid, And Antioxidant Capacity of Noni (Morinda citrifolia L.) Juice and Powder as Affected by Illumination During Storage," Food Chemistry, Vol. 122, No. 3, 2010, pp. 627-632. doi:10.1016/i.foodchem.2010.03.022

[17] C. Carr and B. Feri, "Toward a New Recommended Dietary Allowance for Vitamin C Based on Antioxidant and Health Effects in Humans," The Americal Journal of Clinical Nutrition, Vol. 69, No. 6, 1999, pp. 1086-1107.

[18] S. Shinkai, S. Mori, H. Koreishi, T. Tsubaki and O. Manabe, "Hexasulfonated Calix[ 6larene Derivatives: A New Class of Catalysts, Surfactants, and Host Molecules," Journal of the American Chemical Society, Vol. 108, No. 9, 1986, pp. 2409-2416. doi: $10.1021 / \mathrm{ja} 00269 \mathrm{a} 045$

[19] Y. Liu, B. H. Han and Y. T. Chen, "Molecular Recognition and Complexation Thermodynamics of Dye Guest Molecules by Modified Cyclodextrins and Calixarenesulfonates," The Journal of Physical Chemistry B, Vol. 106, No. 18, 2002, pp. 4678-4687. doi:10.1021/jp015603r
[20] Y. Y Zhou, H. W. Xu, L. Wu, C. Liu, Q. Lu and L. Wang, "Spectrofluorimetric Study on the Inclusion Interaction Between Vitamin $\mathrm{K}_{3}$ with $p$-( $p$-Sulfonated Benzeneazo) Calix[6]arene and Determination of $\mathrm{VK}_{3}$," Spectrochimica Acta Part A, Vol. 71, No. 2, 2008, pp. 597-602. doi:10.1016/i.saa.2007.11.036

[21] M. Catarina, et al., "Multimodal Molecular Encapsulation of Nicardipine Hydrochloride by $\beta$-Cyclodextrin, Hydroxypropyl-B-Cyclodextrin and Triacetyl-B-Cyclodextrin in Solution. Structural Studies by ${ }^{1} \mathrm{H}$ NMR and ROESY Experiments," European Journal of Pharmaceutical Sciences, Vol. 18, No. 5, 2003, pp. 285-296. doi:10.1016/S0928-0987(03)00025-3

[22] A. L. Thakkarand P. V. Demarco, "Cycloheptaamylose Inclusion Complexes Of Barbiturates: Correlation Between Proton Magnetic Resonance and Solubility Studies," Journal of Pharmaceutical Sciences, Vol. 60, No. 4, 1971, pp. 652-653. doi:10.1002/jps. 2600600444

[23] S. Shinkai, "Calixarenes-The Third Generation Of Supramolecules," Tetrahedron, Vol. 49, No. 40, 1993, pp. 8933-8968. doi:10.1016/S0040-4020(01)91215-3

[24] Y. Y. Zhou, C. Liu, H. W. Xu, H. P. Yu, Q. Lu and L. Wang, "Study on the Fluorescence Behavior of $p$-Sulfonated Calix[4,6]arene in Cationic Surfactant Cetyltrimethylammonium Bromide Solution and Its Analytical Application," Spectrochimica Acta Part A, Vol. 66, No. 4-5, 2007, pp. 919-923. doi:10.1016/j.saa.2006.04.031

[25] C. Liu, Z. Fu, H. P. Yu, H. W. Xu, L. Wang and Y. Y. Zhou, "Spectrofluorimetric Study on the Inclusion Behavior of p-Sulfonated Calix[6]arene with Cetyltrimethylammonium Bromide and Analytical Application," Journal of Luminescence, Vol. 126, No. 2, 2007, pp. 747-752. doi:10.1016/j.jlumin.2006.11.006 